\title{
Relación entre bullying y conductas suicidas en adolescentes: una revisión sistemática
}

\author{
Gonzalo Galván ${ }^{1}$, Lina María Castilla Chaverra ${ }^{1}$, Marje de Jesús Garces Ricardo ${ }^{1}$, \\ Francisco Javier Vásquez De la Hoz ${ }^{1}$, Manuel Francisco Guerrero Martelo', \\ Laura Juliana Barchelot Aceros ${ }^{2}$, Jairo Torres Oviedo ${ }^{3}$
}

\begin{abstract}
1. Universidad Cooperativa de Colombia. Montería, Córdoba, Colombia.
2. Universidad de Investigación y Desarrollo (UDI), Bucaramanga, Santander, Colombia.

3. Universidad de Córdoba. Montería, Córdoba, Colombia.
\end{abstract}

Autor correspondiente: Gonzalo Galván, E-mail: galvan.patrignani@gmail.com

\begin{abstract}
Resumen
Introducción: la relación entre bullying y comportamiento suicida (CS) adolescente se manifiesta por altos índices de intentos o suicidios consumados en víctimas de maltrato. A pesar de la importancia del tema son pocos los estudios que dan cuenta de los pormenores de la relación entre estas variables lo que dificulta la crear intervenciones eficaces. El objetivo de este estudio fue proporcionar una visión más detallada acerca de la relación entre bullying y CS. Método: Revisión sistemática de literatura incluyendo publicaciones de índices bibliográficos, ScienceDirect y Pubmed utilizando los términos "bullying" AND "suicidal behavior" AND "adolescents" AND "school harassment" AND "school mistreatment" y sus correspondientes en español publicados desde 2012 hasta 2018. Resultados: se incluyeron 21 artículos de 272 iniciales que muestran los pormenores de la relación entre bullying y comportamiento suicida: edad, género, tipos y frecuencias de victimización (verbal, física, relacional, cyberbullying, sexual y polivictimización) y su relación con variables del CS (ideación, planificación, intentos y suicidio consumado). Conclusiones: comunicar que solo hecho de recibir bullying resulta en CS minimiza la existencia de otros factores moduladores y empeora el entendimiento de la relación entre los fenómenos conduciendo a una negligencia en potenciales acciones que pudieran llevarse a cabo en intervención. Palabras clave: Bullying - Comportamiento suicida - Adolescentes - Acoso escolar - Maltrato escolar.
\end{abstract}

\section{RELATIONSHIP BETWEEN BULLYING AND SUICIDAL BEHAVIORS IN ADOLESCENTS: A SYSTEMATIC REVIEW}

\begin{abstract}
Introduction: The relationship between bullying and adolescent suicidal behavior (SB) is manifested by high rates of attempted or committed suicide in victims of abuse. In spite of the importance of the topic, there are few studies that explain the details of the relationship between these variables, which makes it difficult to create effective interventions. The objective of this study was to provide a more detailed view about the relationship between bullying and SB. Method: Systematic review of literature including publications of bibliographic indexes, ScienceDirect and Pubmed using the terms "bullying" AND "suicidal behavior" AND "adolescents" AND "school harassment" AND "school mistreatment" and their corresponding in Spanish published from 2012 to 2018. Results: 21 articles of 272 initials that show the details of the relationship between bullying and suicidal behavior were included: age, gender, types and frequencies of victimization (verbal, physical, relational, cyberbullying, sexual and poly-victimization) and their relationship with variables of SB (ideation, planning, attempts and consummate suicide). Conclusions: Communicate that only receiving bullying results in SB minimizes the existence of other modulating factors and worsens the understanding of the relationship between phenomena leading to negligence in potential actions that could be carried out in intervention.
\end{abstract}

Key words: Bullying - Suicidal behavior - Adolescents - School harassment - School mistreatment. 


\section{Introducción}

El bullying es un fenómeno conductual que ha acompañado a la sociedad desde sus inicios hasta nuestros días (1) y se entiende como la dinámica de maltrato intencional y sistemática que ocurre entre iguales de manera persistente (2). Los investigadores han identificado en este tipo de actos una serie de intimidaciones desfavorables de tipo físicas, verbales y psicológicas $(3,4)$ que son repetitivas, causando un malestar significativo a la víctima y estableciendo un desequilibrio de dominio entre ella y su acosador (5).

El bullying es un problema que a nivel global existe en diferentes situaciones y formas en todos los países. UNICEF en 2014 extrajo datos sobre el bullying en 106 países y los resultados indicaron lo siguiente: la proporción de adolescentes, en edades entre 13 y 15 años, que alguna vez han experimentado bullying, va desde el $7 \%$ en Tayikistán hasta el 74\% en Samoa. Entre los países de alto poder adquisitivo de los que existen datos, el rango estaría entre el 9\% en Italia y el 52\% en Lituania. En 14 de los 67 países de bajo y medio poder adquisitivo con datos, más de la mitad de la población estudiantil expresó haber sufrido acoso recientemente. Por otro lado, una parte importante de los adolescentes de Europa y América del Norte admitieron haber acosado a otros, con una prevalencia que va desde el 14\% (1 de 7) en la República Checa y Suecia, hasta casi el 59\% (6 de 10) en Letonia y Rumania (5).

Diferentes investigaciones afirman que algunas personas que son víctimas de bullying contarían con características específicas, tales como una discapacidad física e intelectual o por destacarse en el ámbito académico a los que por lo regular se les denomina "los dejados", "puerquitos", "maricones", "rajones", "chillones", ente otros adjetivos que se utilizan dentro de las instituciones $(6,7)$.

Por otra parte, el bullying se configura como un estresor vital $(8,9)$ que daría cuenta de una relación cruel de dominio-sumisión que cuando se practica de manera recurrente da lugar a un proceso de victimización, llevando con él un deterioro psicológico de la personalidad de la víctima y un deterioro moral del agresor. Sin embargo, es a la víctima a quien le puede traer consecuencias más severas, debido a que su situación puede terminar tanto en fracaso académico como en un daño físico o psicológico e consecuencias más graves (10). El maltrato puede generar en las víctimas sentimiento de desprotección y humillación, aislamiento, depresión, neurosis e histerias, auto-concepto negativo, incremento en el estado de ansiedad, fobias a las instituciones educativas y a todo el entorno escolar y reacciones agresivas $(11,12,8,9)$.

De acuerdo a lo anterior, y entre las consecuencias potenciales de recibir bullying la más crítica es la configurada por la muerte evidentemente autoinflingida por medio de la utilización de cualquier método es decir, el suicidio (13). Según diferentes informes e investigaciones el suicidio es la cuarta causa de muerte entre los jóvenes de 10 a 19 años de edad $(14,15,16)$ y la segunda causa entre los 15 y 29 años (17). Particularmente en Colombia, en 2015, se presentaron en Colombia 2.068 suicidios, 10 $\%$ más que en el año anterior, 48,74 \% de ellos en el grupo de edad de 15 a 34 años, con mayor participación de jóvenes entre 20 y 24 años (14,60\%); en el rango de edad de los 10 a los 14 años, la tasa por 100.000 habitantes fue de 1,73, y en el de 15 a 17 años, de 5,72 por 100.000 habitantes (8). Preocupa que en Colombia, entre el 2005 y el 2009, se presentó una tasa del 0,07 entre los niños de 5 a 9 años y del 4,74 entre los de 10 y 19 años pues desde tempranas edades, niños y adolescentes, estén considerando la muerte como una opción para la solución de sus problemas y conflictos (18).

Tanto el bullying como el comportamiento suicida representan problemas de salud pública $(19,20)$ y la relación entre ambas variables implica no solo a aquellos que reciben maltrato, sino también a maltratadores y a quienes han estado en ambos roles.

La asociación entre bullying y comportamientos suicidas es al menos compleja. Los hallazgos de estudios, sobre todo longitudinales, indican que existe un incremento del riesgo de ideación e intentos suicidas en las personas que reciben bullying pero que esa relación es al menos variable y se encuentra modulada por otras variables (21). Comunicar que el solo hecho de recibir bullying resulta en comportamiento suicida minimiza la existencia de otros factores moduladores y empeora el entendimiento de la relación entre los fenómenos conduciendo a una negligencia de las potenciales acciones que pudieran llevarse a cabo para la intervención (19).

\section{Materiales y método}

En la elaboración de esta revisión sistemática se siguieron recomendaciones del Prisma Group para la publicación de este tipo de estudios. Durante las últimas décadas, se han llevado a cabo importantes iniciativas para mejorar la transparencia, la calidad y la consistencia de la información metodológica y los resultados presentados en las revisiones sistemáticas y metaanálisis. Entre ellas, destaca la publicación en 1999 de la declaración Quality of Reporting of Meta-analyses -QUOROM-, y su posterior revisión y ampliación en la declaración Preferred Reporting Items for Systematic Reviews and Meta-Analyses (22).

\section{Selección de los estudios}

Se seleccionaron publicaciones de revistas científicas (en inglés y español) exclusivamente (no se incluyeron tesis doctorales ni capítulos de libro) centradas en estudios empíricos sobre relación entre el suicidio en adolescentes y el bullying. Los artículos fueron extraídos de datos de la población general a través de los índices bibliográficos ScienceDirect, Pubmend utilizando los siguientes términos "suicidal behavior" AND "bullying" AND "adolescents" AND "school harassment" AND "school mistreatment" y sus correspondientes en español "comportamiento suicida" AND "adolescentes" AND "hostigamiento escolar" AND "maltrato escolar" publicados desde 2012 hasta 2018 , incluido. 


\section{Criterios de inclusión y exclusión}

\section{Inclusión}

Para ser incluidos en esta revisión, los estudios debían cumplir los siguientes criterios: a) centrarse en la evaluación y estudio de una muestra de adolescentes entre 12 a 19 años; b) aportar datos de prevalencia tanto de victimas de bullying como de comportamiento suicida; c) respecto a la prevalencia de comportamiento suicida, aportar datos sobre ideación suicida, planificación suicida y/o intentos de suicidio; d) evaluar el riesgo de suicidio a partir de la experiencia de bullying; y e) artículos en inglés y español que se encuentren dentro del rango de tiempo de 2012 a 2018.

\section{Exclusión}

Como criterios de exclusión, se excluyeron todos aquellos estudios que: a) no incluyeran los temas de la revisión como el bullying, comportamientos suicidas y la relación entre ambas; b) evaluaran muestra clínica o población judicializada; c) estén fuera del rango temporal estipulado; d) evaluaran solo la prevalencia de bullying y suicidio, pero sin analizar la relación entre ellos o, e) fueran artículos de revisión.

\section{Codificación de los estudios}

Se registraron una serie de variables relativas a los trabajos revisados relacionados con el diseño y la metodología del estudio: a) autores y año de publicación; b) tipo de institución educativa (pública, privada, religiosa, laica): c) número de participantes; d) origen de la muestra; e) sexo de los menores (varones, mujeres, ambos sexos); f) rango de edad de los participantes (en años) o edad media o cursos a los que asistían; g) prevalencia de bullying; y h) definición del tipo de bullying experimentado (psicológico o físico). A su vez, se registraron variables relacionadas con comportamiento suicida en adolescentes víctimas de bullying: i) prevalencia de ideación suicida; j) prevalencia de planificación suicida; k) prevalencia de intentos de suicidio; y l) variables asociadas al bullying y a la conducta suicida.

\section{Resultados}

En la siguiente figura (Fig. 1) se puede observar el proceso por el cual fueron seleccionaron los artículos de la presente revisión. Tras la búsqueda inicial (272 registros) se incluyeron 21 registros, excluyendo 251 por no cumplir con los criterios de inclusión/exclusión anteriormente detallados. No hubo registros duplicados.

\section{Figura 1.}

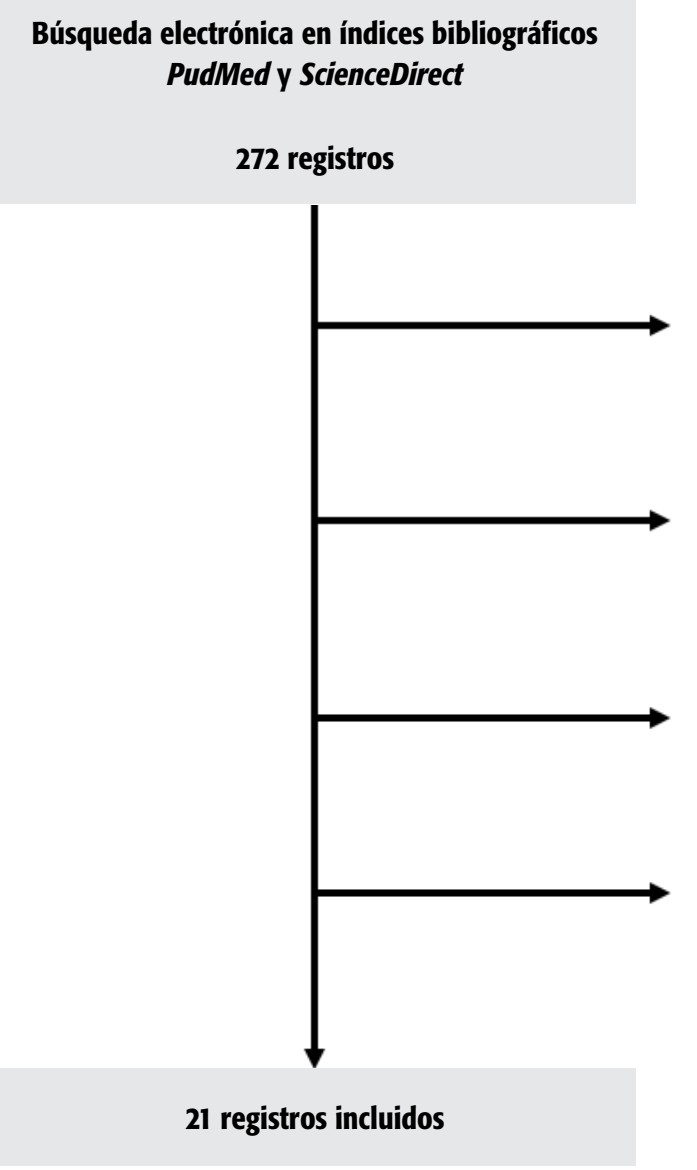

51 registros excluidos por basarse en muestra clínica o en un colectivo específico

20 registros excluidos por ser artículos de revisión

0 registros duplicados

180 registros excluidos por no cumplir criterios 


\section{Variables relacionadas con el diseño y la metodolo- gía de los estudios}

Como se observa en el siguiente cuadro (Cuadro 1), los estudios que conforman la muestra final de esta revisión proporcionaron información sobre la relación entre el bullying y las conductas suicidas en adolescentes, recogiendo datos originales de 17 países diferentes entre los años 2012 y 2018. La mayoría de publicaciones provienen de USA (61.9\%), seguida de Europa (9.5\%), China (9.5\%), Ghana (4.7\%), Países de Oriente (4.7\%), Canadá (4.7\%) y Hawái (4.7\%). No se hallaron estudios en países latinoamericanos.

Con respecto al tipo de muestreo utilizado en el proceso de selección de los participantes, en un $71.4 \%$ se utilizaron métodos probabilísticos de obtención de la muestra, donde $64.3 \%$ son probabilísticos aleatorios y $35.7 \%$ son probabilísticos estratificados; en el $28.6 \%$ restante de las investigaciones se utilizó muestreo no probabilístico. Los participantes de las muestras fueron reclutados a partir de encuestas realizadas en instituciones educativas ( $\mathrm{n}=66,7 \%)$ por un lado $\mathrm{y}$, por otro lado, fueron reclutados a través de estudios llevados a cabo en la población general $(n=33.3 \%)$.

El rango de los tamaños muéstrales utilizados en los estudios fue amplio ya que oscilo entre 96 y 130.908 sujetos.

Variables relacionadas con los participantes y con el tipo de victimización experimentada

La participación por género en los estudios mostró un porcentaje similar de varones y mujeres $(48,16 \%$ vs $51,70 \%$, respectivamente). Las muestras de los estudios seleccionados comprendieron un rango de edades situados entre los 10 y 24 años, 4 estudios no describieron edad media ni rango de edad de los participantes, sin embargo, mostraron los grados académicos a los que asistían que estuvieron situados entre $6^{\circ}$ a $12^{\circ}$.

En cuanto a las variables relacionadas a la victimización del bullying, en los estudios incluidos en esta revisión destacan (en orden decreciente de prevalencia): la victimización verbal (71.4\%), victimización física $(52.3 \%)$, bullying relacional o baja conexión social (23.8\%), cyberbullying $23.8 \%$, bullying sexual (9.5\%), poli-victimización $(4.7 \%)$, entre otras. Cabe resaltar que la suma de porcentajes no equivale al $100 \%$ dado que las mismas variables son comunes a diversos artículos.

\section{Variables relacionadas con el riesgo de comporta- miento suicida en víctimas de bullying}

La mayor parte de los estudios (80.9\%), consultados ofrecieron datos sobre el riesgo de ideación suicida y/o intentos suicidas, siendo menor el número de estudios que aportaron datos sobre planificación suicida (14.2\%). Un $4.7 \%$ de los estudios indicaron datos generales de comportamiento suicida sin diferenciar entre de ideación, planificación o intentos de suicidios. En cuanto a la muestra total de los estudios incluidos ( $\mathrm{N}=253.770)$, 184.204 sujetos presentaron ideación suicida, 792 intento de suicidio, 313 planificación suicida y 196 suicidio consumado.

En relación a las variables relacionadas a la experiencia de la victimización de bullying, los estudios incluidos permiten reflejar la relación de los tipos de bullying con ideación, planificación e intentos de suicidio. Los sujetos que habían recibido sólo bullying verbal (71.4\%) comparados con los que habían sufrido sólo maltrato, presentaron mayor riesgo de planificación suicida, debido al rechazo al que eran expuesto por sus compañeros. Los adolescentes víctimas de bullying físico (52.3\%), por parte de sus compañeros de escuela, tenían un mayor riesgo de presentar intentos de suicidio que los que no habían sufrido este tipo de bullying, y esto era independiente de si habían sufrido maltrato en la infancia o no, debido a que los efectos del maltrato infantil sobre la salud mental en la etapa adulta joven, estuvieron parcialmente relacionados con el haber sufrido bullying en la infancia.

Por otra parte, los adolescentes que se sintieron rechazados por sus parejas (23.8\%) y no se llevaban bien con sus compañeros en el colegio, presentaron un mayor riesgo de ideación suicida en el futuro que los que sí tenían buenas relaciones en el colegio y con sus parejas. Aquellos adolescentes que fueron víctimas de $c y$ berbullying (23.8\%) mostraron niveles incrementados de intentos de suicidio comparados con otras personas del mismo sexo en diferentes tipos de victimización, lo que los cataloga como un grupo vulnerable, debido a que es realizado en el anonimato y por la falta de control que presenta la víctima ante el victimario. Los jóvenes que reportaron bullying sexual (9.5\%) desarrollaron intentos de suicidio posterior a la victimización, presentando de igual manera problemas de depresión por el rechazo recibido por sus parejas. Los adolescentes que sufrían poli-victimización $(4.7 \%)$ y tenían algún otro factor de riesgo suicida como depresión, eran los que presentaban un mayor riesgo de reportar ideación suicida al poco tiempo de haber sufrido maltrato comparados con adolescentes sin antecedentes de bullying, pero con otros factores de riesgo para conducta suicida. Por último, también es importante tener en cuenta las diferencias de género que se evidencian en los estudios, las mujeres presentan un nivel más alto de depresión e ideación suicida que los hombres, como consecuencia de la constante victimización verbal y cibernética.

\section{Discusión}

La revisión realizada tuvo por objetivo identificar las relaciones entre bullying y las conductas suicidas en adolescentes a nivel mundial, siendo ambos problemas de salud pública con un alto nivel de afectación en la sociedad y principalmente entre los jóvenes. Se analizaron las variables relacionadas a estas problemáticas, como lo es la victimización física y verbal, cyberbullying, bullying sexual, poli-victimización y victimización en las rela- 
Características de los estudios incluidos en la revisión

\begin{tabular}{|c|c|c|c|c|c|}
\hline Autores y año & País de la muestra & $\mathbf{N}$ & Muestreo & $\begin{array}{l}\text { Origen de la } \\
\text { muestra }\end{array}$ & $\begin{array}{l}\text { Rango de edad/ } \\
\text { Edad media o Cursos } \\
\text { a los que asisten }\end{array}$ \\
\hline $\begin{array}{l}\text { Fredrick \& Demaray, } 2018 \\
(23)\end{array}$ & EEUU & 403 & No probabilístico & Estudiantes & $\begin{array}{l}9^{\circ} \text { grado } \\
(13 \text { a } 16 \text { años })\end{array}$ \\
\hline Barzilay, y otros, 2017 (24) & $\begin{array}{c}\text { Europa (Austria, Estonia, } \\
\text { Francia, Alemania, Hungría, } \\
\text { Irlanda, Italia, Rumania, } \\
\text { Eslovenia, España) }\end{array}$ & 11.110 & Randomizado & Estudiantes & 15 años \\
\hline $\begin{array}{l}\text { Baiden, Stewart, \& Fallon, } \\
2017(25)\end{array}$ & Ontario, Canadá & 1650 & No probabilístico & Estudiantes & 12 a 18 años \\
\hline $\begin{array}{l}\text { Arango, Opperman, } \\
\text { Gipson, \& King, } 2016 \text { (26) }\end{array}$ & $\begin{array}{l}\text { Región del medio oeste - } \\
\text { EEUU }\end{array}$ & 321 & $\begin{array}{l}\text { Probabilístico: } \\
\text { aleatorio }\end{array}$ & Población general & $\begin{array}{l}12 \text { a } 15 \text { años } \\
\text { (13.6 media) }\end{array}$ \\
\hline Hong, y otros, 2016 (27) & Provincia de Fujian de China & 20.509 & $\begin{array}{l}\text { Probabilístico: } \\
\text { estratificado }\end{array}$ & Estudiantes & $16.3(\mathrm{DE} 2.0)$ \\
\hline Rodway, y otros, 2016 (28) & Inglaterra & 130 & $\begin{array}{l}\text { Probabilístico: } \\
\text { aleatorio }\end{array}$ & $\begin{array}{l}\text { Población clínica: } \\
\text { registros de } \\
\text { decesos }\end{array}$ & 10 a 19 años \\
\hline $\begin{array}{l}\text { Sibold, Edwards, Murray- } \\
\text { Close, \& Hudziak, } 2015 \\
\text { (29) }\end{array}$ & EE.UU. & 13.583 & No probabilístico & Estudiantes & $\begin{array}{l}\text { Grados } 9^{\circ} \text { a } 12^{\circ} \\
\text { edades comprendidas } \\
\leq 14 \mathrm{a} \geq 18 \text { años }\end{array}$ \\
\hline $\begin{array}{l}\text { Ohene, Johnson, Atunah- } \\
\text { Jay, Owusu, \& Borowsky, } \\
2015 \text { (30) }\end{array}$ & Ghana & 1.984 & No probabilístico & Estudiantes & $12 \mathrm{a} \geq 18$ años \\
\hline $\begin{array}{l}\text { Bhatta, Shakya, \& Jefferis, } \\
2014 \text { (31) }\end{array}$ & $\begin{array}{l}\text { Condado rural de los } \\
\text { Apalaches en Ohio - EE. UU. }\end{array}$ & 468 & $\begin{array}{l}\text { Probabilístico: } \\
\text { aleatorio }\end{array}$ & Población general & 11 a 15 años \\
\hline $\begin{array}{l}\text { Messias, Kindrick, \& } \\
\text { Castro, } 2014(32)\end{array}$ & EE.UU. & 15.425 & No Probabilístico & Estudiantes & 14 a 18 años \\
\hline $\begin{array}{l}\text { Zimmerman \& Posick, } \\
2014 \text { (33) }\end{array}$ & Chicago & 1.628 & $\begin{array}{l}\text { Probabilístico: } \\
\text { aleatorio }\end{array}$ & Población general & 14.8 (DE. 2.5) años \\
\hline $\begin{array}{l}\text { Dunn, Gjelsvik, Pearlman, } \\
\text { \& Clark, } 2014 \text { (34) }\end{array}$ & EE.UU. & 13.065 & $\begin{array}{l}\text { Probabilístico: } \\
\text { estratificado }\end{array}$ & Estudiantes & Grado $9^{\circ}$ a $12^{\circ}$ \\
\hline Henry, y otros, 2014 (35) & Noreste de EE. UU. & 2.936 & $\begin{array}{l}\text { Probabilístico: } \\
\text { aleatorio }\end{array}$ & Estudiantes & Grado de $6^{\circ}$ a $12^{\circ}$ \\
\hline $\begin{array}{l}\text { Litwiller \& Brausch, } 2013 \\
\text { (36) }\end{array}$ & EE.UU. & 4.693 & $\begin{array}{l}\text { Probabilístico: } \\
\text { estratificado }\end{array}$ & Estudiantes & 16.11 (DE: 1.20) \\
\hline $\begin{array}{l}\text { Bauman, Toomey, \& } \\
\text { Walker, } 2013 \text { (37) }\end{array}$ & Hawai & 1.491 & $\begin{array}{l}\text { Probabilístico: } \\
\text { aleatorio }\end{array}$ & Estudiantes & Grado de 9 a 12 \\
\hline $\begin{array}{l}\text { Turner, Exum, Brame, \& } \\
\text { Holt, } 2013(38)\end{array}$ & Carolina del Norte central & 1.874 & $\begin{array}{l}\text { Probabilístico: } \\
\text { aleatorio }\end{array}$ & Población general & $\begin{array}{c}\text { Grados } 6 \text { a } 12 \\
\text { (edad media 13.77) }\end{array}$ \\
\hline $\begin{array}{l}\text { Borowsky, Taliaferro, \& } \\
\text { McMorris, } 2013 \text { (39) }\end{array}$ & Minnesota & 130.908 & $\begin{array}{l}\text { Probabilístico: } \\
\text { aleatorio }\end{array}$ & Estudiantes & Grados $6^{\circ}, 9^{\circ}$ y $12^{\circ}$ \\
\hline Chan, 2013 (40) & China & 18.341 & $\begin{array}{l}\text { Probabilístico: } \\
\text { estratificado }\end{array}$ & Población general & 15 a 17 años \\
\hline Lereya, y otros, 2013 (41) & EE.UU. & 481 & $\begin{array}{l}\text { Probabilístico: } \\
\text { aleatorio }\end{array}$ & Población general & 7 y los 17 años \\
\hline Klomek, y otros, 2013 (42) & EE.UU. & 96 & $\begin{array}{l}\text { Probabilístico: } \\
\text { estratificado }\end{array}$ & Estudiantes & 13 a 18 años \\
\hline $\begin{array}{l}\text { Harel-Fisch, Abdeen, } \\
\text { Walsh, Radwan, \& Fogel- } \\
\text { Grinvald, } 2012 \text { (43) }\end{array}$ & $\begin{array}{l}\text { Medio Oriente: judíos / } \\
\text { árabes israelíes; Palestinos } \\
\text { de Cisjordania }\end{array}$ & 8.345 & $\begin{array}{l}\text { Probabilístico: } \\
\text { estratificado }\end{array}$ & Estudiantes & 15 años \\
\hline
\end{tabular}


ciones sociales $(44,45)$. Como se pudo constatar en este trabajo, el suicidio se encuentra presente en gran parte de las víctimas que sufren cualquier tipo de bullying, por lo que se considera que la relación entre bullying y las conductas suicidas es relevante.

Si bien los artículos consultados describen una alta variabilidad con respecto a la ideación suicida e intentos de suicidio, en general las victimas de bullying durante la etapa de la adolescencia tienen un riesgo mayor de presentar conductas suicidas. Es importante destacar que los estudios indican que entre los adolescentes que recibieron bullying las mujeres son más propensas a presentar un nivel más alto de ideación suicida, no obstante, en el género masculino se presentan casos más frecuentes de suicidio consumado.

A nivel metodológico, la mayor parte de las investigaciones consultadas son provenientes de Estados Unidos siendo escasos los países orientales, latinos y europeos que se han tomado la tarea de realizar este tipo de investigación. Sin embargo, es importante resaltar que fueron varios los estudios realizados acerca del tema en Latinoamérica, pero no cumplían con los criterios de inclusión establecidos, no tenían la información requerida como los tipos de bullying, datos generales de ideación, planificación e intentos de suicidio y la relación entre bullying-suicidio.

En cuanto a los estudios seleccionados se resalta como aspecto positivo, especificar el uso de métodos probabilísticos para la escogencia de las muestras en la gran mayoría de los trabajos, el uso de las muestras en una dimensión importante, con una cantidad que va desde 96 hasta a más de 1000 participantes en la gran mayoría de las investigaciones consultadas, así como la incorporación de ambos géneros con un porcentaje más o menos similar en la totalidad de las investigaciones, apunta a una mayor posibilidad de validez y confiabilidad en los resultados obtenidos y una mayor probabilidad de generalización de los mismos.

Por otro lado, la mayoría de la información acerca de la relación entre el bullying y las conductas suicidas proviene de estudios transversales, lo que ha limitado el poder establecer una verdadera relación de causalidad (10).

Cabe resaltar que son cuantiosos los estudios que asocian las variables suicidio y bullying en adolescentes, pero muy contadas las que explican la relación que existe entre ellas. La victimización verbal, la victimización física, el bullying relacional o baja conexión social, el cyberbullying, el bullying sexual y la poli-victimización se observan en un alto número de casos, como también se evidencia en los trabajos que validan las consecuencias y la repercusión que pueden tener a corto y largo plazo en adolescentes víctimas de bullying. Entre las escasas investigaciones que han estudiado este tema, muchas indican que la falta de apoyo familiar y social son factores relevantes en la relación bullying-suicidio; el escaso apoyo familiar parece encontrarse muy inmerso en esta temática, debido a que es un factor de riesgo bastante importante en estos adolescentes, por ser una red de apoyo ausente en donde ellos esperan encontrar un soporte primordial y no es hallado de la manera que ellos esperan.

Las investigaciones recientes han demostrado que los niños y adolescentes que sufren de bullying manifiestan problemas de adaptación, dificultades sociales, problemas físicos y conductas suicidas, lo cual permite realizar una asociación entre el bullying y las conductas suicidas, las cuales parten del impacto y la frecuencia así como del tipo de victimización al que se está siendo sometido, ya que ser víctima concurrente se asocia a conductas suicidas posteriores $(46,47)$. En cuanto a los tipos de bullying (ya sea físico, verbal, cyberbullying, por baja conexión social, bullying sexual y poli-victimización) si estos se presentan de forma frecuente se podría conducir a las víctimas a tener baja autoestima, una actitud pasiva fuera de lo normal, falta de interés por los estudios y bajo rendimiento académico; así mismo, pueden alterar la conducta de la víctima, generando intromisión, introversión, timidez, aislamiento social y soledad, lo cual puede ser causa de síntomas depresivos y de ideación suicida (48). Los adolescentes que sufren los tipos de bullying descritos, por sentirse inferiores al agresor, no se sienten en la capacidad de enfrentar la situación o buscar ayuda en otras personas, por ende, las víctimas de bullying tienen el pensamiento de que son un estorbo para la sociedad, no sirven para nada, etc. De igual forma, el ser maltratado o golpeado constantemente, y ser sometido a comentarios ofensivos y burlas, conlleva a que los adolescentes se aíslen, abandonen la escuela, presenten poco interés por las actividades que realizaban antes y busquen espacios donde se encuentren en completa soledad. En el caso particular del $c y$ berbullying (el cual es una nueva forma de realizar victimización por medio de las redes sociales) los agresores tienen la oportunidad y facilidad durante las 24 horas del día de mantener a su víctima sometida a constantes amenazas, publicando su vida íntima, creando imágenes o videos burlescos sobre su apariencia física y por su personalidad, ocasionando en la víctima sentimientos de tristeza, depresión y ansiedad, el sentimiento de culpabilidad, y en última instancia, la aparición de pensamientos suicidas e incluso intentar acabar con sus vidas.

Por último, por sufrir bullying sexual, la poli-victimización y la victimización por baja conexión social, se puede presentar depresión, baja autoestima, desesperanza e incluso el consumo de sustancias psicoactivas y alcoholismo, sentimientos de inutilidad y de culpa; pero es necesario más investigaciones que evalúen los factores de personalidad, teniendo en cuenta que estos pueden generar trastornos psicopatológicos generando un alto riesgo de conducta suicida (49).

\section{Conclusiones}

En conclusión, los resultados obtenidos de las investigaciones, muestran una importante cantidad de adolescentes que han sido víctimas de bullying por parte de su entorno, ya sea en las instituciones educativas o 
en su entorno social; y como consecuencia de esto, van adquiriendo pensamientos y conductas suicidas, siendo conveniente un mayor conocimiento sobre los efectos que estas conductas puedan tener a corto y largo plazo, teniendo en cuenta la importancia de indagar más sobre la intensidad y presencia de estos pensamientos suicidas y de qué tanto riesgo corren las víctimas en llevar a cabo la conducta como consecuencia de la victimización.

En cuanto a lo anterior, se considera que el conocimiento de los resultados aquí expuestos, pueden posibilitar y encaminar a una intervención más apropiada de los adolescentes que sufren de este tipo de victimización, así como el planteamiento de actividades de promoción y prevención de estas conductas, que se enfoquen en el alto riesgo que significa la práctica del bullying en adolescentes y las consecuencias que desencadenan este tipo de conductas, tanto a nivel cognitivo como comportamental, que permita prevenir los problemas de baja autoestima, depresión, bajo rendimiento académico, falta de interés por sus actividades diarias, aislamiento social, introversión, timidez y síntomas depresivos que lo lleven a tener ideación, planificación e intentos de suicido, debido a que estas son las consecuencias de la relación que entrelazan al bullying y a los pensamientos y las conductas suicidas, que sean eficientes y claros para poder mitigar esta conducta.

\section{Conflictos de interés}

Los autores confirman no tener conflicto de interés respecto de este artículo.

\section{Referencias bibliográficas}

1. Salas Picón WM. Revisión sobre la definición del bullying. Revista Poiésis. 2015; 30: p. 44-50.

2. Musalem B, Castro P. Qué se sabe de bullying. Revista Médica Clínica Las Condes. 2015; 26(1): p. 14-23.

3. Albores-Gallo L, Sauceda-García JM, Ruiz-Velasco S, Roque-Santiago E. El acoso escolar (bullying) y su asociación con trastornos psiquiátricos en una muestra de escolares en México. Salud Pública de México. 2011; 53(3): p. 220227.

4. Lugones Botell M, Ramírez Bermúdez M. Bullying: aspectos históricos, culturales y sus consecuencias para la salud. Revista Cubana de Medicina General Integral. 2017; 33(1): p. 154-162.

5. UNICEF. Ocultos a plena luz. Un análisis estadístico de la violencia contra los niños. Resumen; 2014.

6. Valdebenito S, Ttofi MM, Eisner M, Gaffney H. Weapon carrying in and out of school among pure bullies, pure victims and bully-victims: A systematic review and meta-analysis of cross-sectional and longitudinal studies. Aggression and Violent Behavior. 2017; 33: p. 62-77.

7. Aldridge JM, McChesney K. The relationships between school climate and adolescent mental health and wellbeing: A systematic literature review. International Journal of Educational Research. 2018; 88(1): p. 121-145.

8. Montoya-Gómez B. Comportamiento del suicidio. Colombia, 2015. Violencia autoinigida, desde un enfoque forense. ; 2015.

9. Carballo Belloso J, Gómez Peñalver J. Relación entre el bullying, autolesiones, ideación suicida e intentos autolíticos en niños y adolescentes. Revista de Estudios de Juventud. 2017;(115): p. 207-218.

10. van Geel M, Goemans A, Vedder P. A meta-analysis on the relation between peer victimization and adolescent non-suicidal self-injury. Psychiatry Research. 2015; 230(2): p. 364-368.

11. Contreras Álvarez ÁP. El fenómeno de bullying en Colombia. Revista Logos, Ciencia \& Tecnología. 2013; 4(2): p. 100114.

12. Le H, Tran N, Campbell M, Gatton M, Nguyen H, Dunne M. Mental health problems both precede and follow bullying among adolescents and the effects differ by gender: a cross-lagged panel analysis of school-based longitudinal data in Vietnam. International Journal of Mental Health Systems. 2019; 13(35).

13. Ministerio de Salud y Protección Social. Boletín de salud mental. Conducta suicida. Bogotá:; 2018.

14. Organización Panamericana de la Salud. Mortalidad por suicidio en las Américas. Informe regional. ; 2014.

15. Organización Panamericana de la Salud. Violencia juvenil autoinfligida en América Latina y el Caribe de habla inglesa, 2015.

16. Lagares Franco C, Almenara-Barrios J, O’Ferrall-González C, Castellvi-Obiols P, Gabilondo A, Blasco-Cubedodo MJ, et al. Medidas de frecuencia utilizadas en estudios de cohortes para evaluar el comportamiento suicida en jóvenes (12-26 años): una revisión sistemática. Revista de Psiquiatría y Salud Mental. 2019; 12(4): p. 213-231.

17. Organización Mundial de la Salud. Suicidio. [Online].; 2018. Available from: https://www.who.int/es/news-room/ fact-sheets/detail/suicide.

18. Córdoba RN. Suicidio en niños y adolescentes. Biomédica. 2016; 36(3): p. 341-342.

19. Feldman Hertz M, Donato I, Wright J. Bullying and suicide: a public health approach. Journal of Adolescent Health. 2013; 53.

20. Kowalski R, Limber S. Psychlogical, physical, and academic correlates of. Journal of Adolescent Health. 2013; 53 (1 Suppl).

21. Brunstein Klomek A, Sourander A, Gould M. The association of suicide in childhood to young adulthood: a review of cross sectional and longitudinal research findings. The Canadian Journal of Psychiatry. 2010; 5(55).

22. Hutton B, Catalá-López F, Moher D. La extensión de la declaración PRISMA para revisiones sistemáticas que incorporan metaanálisis en red: PRISMA-NMA. Medicina Clínica. 2016; 147(6): p. 231-280.

23. Fredrick S, Demaray M. Peer victimization and suicidal ideation: The role of gender and depression in a school-based sample. Journal of School Psychology. 2018; 67: p. 1-15.

24. Barzilay S, Klomek A, Apter A, Carli V, Wasserman C, Hadlaczky $\mathrm{G}$, et al. Bullying victimization and suicide ideation and behavior among adolescents in Europe: A 10-country study. Journal of Adolescent Health. 2017; 61(2): p. 179-186. 
25. Baiden P, Stewart S, Fallon B. The mediating effect of depressive symptoms on the relationship between victimization by bullying and non-suicidal self-harm among adolescents: community findings and mental health settings for hospitalized patients in Ontario, Canada. Psychiatry Research. 2017; 255: p. 238-247.

26. Arango A, Opperman K, Gipson P, King C. Suicidal ideation and suicide attempts among youth who report bully victimization, bully perpetration and/or low social connectedness. J Adolesc. 2016; 51: p. 19-29.

27. Hong L, Guo L, Wu H, Li P, Xu Y, Gao X, et al. Bullying, Depression, and Suicidal Ideation Among Adolescents in the Fujian Province of China: A Cross-sectional Study. Medicine. 2016; 95(5): p. e2530.

28. Rodway C, Tham S, Ibrahim S, Turnbull P, Windfuhr $\mathrm{K}$ Shaw J, et al. Suicide in children and young people in England: a consecutive case series. The Lancet Psychiatry. 2016; 3(8): p. 751-759.

29. Sibold J, Edwards E, Murray-Close D, Hudziak J. Physical activity, sadness, and suicidality in bullied US adolescents. Journal of the American Academy of Child \& Adolescent Psychiatry. $2015 ;$ 54(10): p. 808-815.

30. Ohene S, Johnson K, Atunah-Jay S, Owusu A, Borowsky I. Sexual and physical violence victimization among senior high school students in Ghana: Risk and protective factors. Social Science \& Medicine. 2015; 146: p. 266-275.

31. Bhatta M, Shakya S, Jefferis E. Association of being bullied in school with suicide ideation and planning among rural middle school adolescents. Journal of School Health. 2014 84(11): p. 731-738.

32. Messias E, Kindrick K, Castro J. School bullying, cyberbullying, or both: correlates of teen suicidality in the 2011 CDC Youth Risk Behavior Survey. Compr Psychiatry. 2014; 55(5): p. 1063-8.

33. Zimmerman G, Posick C. Detecting specialization in interpersonal violence versus suicidal behavior. Journal of Adolescent Health. 2014; 55(6): p. 810-816.

34. Dunn H, Gjelsvik A, Pearlman D, Clark M. Association between sexual behaviors, bullying victimization and suicidal ideation in a national sample of high school students: implications of a sexual double standard. Women's Health Issues. 2014; 24(5): p. 567-574.

35. Henry K, Lovegrove P, Steger M, Chen P, Cigularov K, Tomazic $\mathrm{R}$. The potential role of meaning in life in the relationship between bullying victimization and suicidal ideation. Journal of Youth and Adolescence. 2014; 43(2): p. 221-232.

36. Litwiller B, Brausch A. Cyber bullying and physical bullying in adolescent suicide: the role of violent behavior and substance use. Journal of Youth and Adolescence. 2013; 42(5): p. 675-84.

37. Bauman S, Toomey R, Walker J. Associations among bullying, cyberbullying, and suicide in high school students. Journal of Adolescence. 2013; 36(2): p. 341-350.

38. Turner M, Exum M, Brame R, Holt T. Bullying victimization and adolescent mental health: General and typological effects across sex. Journal of Criminal Justice. 2013; 41(1): p. 53-59.

39. Borowsky I, Taliaferro L, McMorris B. Suicidal thinking and behavior among youth involved in verbal and social bullying: risk and protective factors. Journal of Adolescent Health. 2013; 53(1): p. S4-S12.

40. Chan KL. Victimization and poly-victimization among school-aged Chinese adolescents: prevalence and associations with health. Preventive Medicine. 2013; 56(3-4): p. 207210.
41. Lereya S, Winsper C, Heron J, Lewis G, Gunnell D, Fisher $\mathrm{H}$, et al. Being bullied during childhood and the prospective pathways to self-harm in late adolescence. Journal of the American Academy of Child \& Adolescent Psychiatry. 2013; 52(6): p. 608-618.e2.

42. Klomek A, Kleinman M, Altschuler E, Marrocco F, Amakawa L, Gould M. Suicidal adolescents' experiences with bullying perpetration and victimization during high school as risk factors for later depression and suicidality. Journal of Adolescent Health. 2013; 53(1): p. S37-S42.43. Harel-Fisch Y, Abdeen Z, Walsh S, Radwan Q, Fogel-Grinvald H. Multiple risk behaviors and suicidal ideation and behavior among Israeli and Palestinian adolescents. Social Science \& Medicine. 2012; 75(1): p. 98-108.

44. Aboujaoude E, Savage MW, Starcevic V, Salame WO. Cyberbullying: Review of an Old ProblemGone Viral. Journal of Adolescent Health. 2015; 57(1): p. 10-18.

45. Halvorsen JA, Lien L, Dalgard F, Bjertness E, Stern RS. Suicidal ideation, mental health problems, and social function in adolescents with eczema: a population-based study. Journal of Investigative Dermatology. 2014; 134(7): p. 1847-1854.

46. Maglione D, Caputi M, Moretti B, Scaini S. Psychopathological consequences of maltreatment among children and adolescents: A systematic review of the GxE literature. Research in Developmental Disabilities. 2018; 82: p. 53-66.

47. Armitage CJ, Panagioti MR, Wirda Abdu R, Rowe R, O'Connor RC. Completed suicides and self-harm in Malaysia: a systematic review. General Hospital Psychiatry. 2015; 37(2): p. 153-165.

48. Upton Patton D, Sung Hong J, Ranney M, Patel S, Kelley C, R. E, et al. Social media as a vector for youth violence: A review of the literature. Computers in Human Behavior. 2014; 35: p. 548-553.

49. Joffre-Velázquez VM, García-Maldonado G, Saldívar-González AH, Martínez-Perales G, Lin-Ochoa D, Quintanar-Martínez $S$, et al. Bullying en alumnos de secundaria. Características generales y factores asociados al riesgo. Boletín Médico del Hospital Infantil de México. 2011; 68(3): p. 193-202.

50. Harrington R. Consecuencias psicosociales de la depresión adolescente. Psiquiatría y Salud Integral. 2001; 1(2): p. 48-52.

51. Sanz García AI,MME. Bullying: What's Going on? A Bibliographic Review of Last Twelve Months. Proced Social and Behavioral Sciences. 2014; 132: p. 269-276.

52. Lereya, S.T.; Muthanna, S.; Wolke, D. Parenting behavior and the risk of becoming a victim and a bully/victim: A meta-analysis study. Child Abuse \& Neglect. 2013; 37(12): p. 1091-1108.

53. Sandoval-Ato R, Vilela-Estrada M, Mejía C, Caballero Alvarado J. Riesgo suicida asociado a bullying y depresión en escolares de secundaria. Revista Chilena de pediatría. 2018; 89(2): p. 208-215

54. Fadanell M, Lemos R, Soto MF, Hiebra MC. Bullying hasta la muerte. Impacto en el suicidio adolescente. Rev Hosp Niños BAires. 2013; 55(249): p. 127 - 135.

55. Kljakovic M, Hunt C. A meta-analysis of predictors of bullying and victimisation in adolescence. Journal of Adolescence. 2016; 49(1): p. 134-145.

56. van Geel M, Vedder P, Tanilon J. Relationship between peer victimization, cyberbullying, and suicide in children and adolescents: a meta-analysis. JAMA Pediatr. 2014; 168(5): p. 435-442.

57. Organización Mundial de la Salud. Plan de Acción sobre Salud Mental 2013-202; 2013 